\title{
Automated Finite Element Analysis for Deformable Registration of Prostate Images
}

\author{
Jessica R. Crouch, Member, IEEE, Stephen M. Pizer, Senior Member, IEEE, Edward L. Chaney, Yu-Chi Hu, \\ Gig S. Mageras, Marco Zaider
}

\begin{abstract}
Two major factors preventing the routine clinical use of finite element analysis for image registration are (1) the substantial labor required to construct a finite element model for an individual patient's anatomy and (2) the difficulty of determining an appropriate set of finite element boundary conditions. This work addresses these issues by presenting algorithms that automatically generate a high quality hexahedral finite element mesh and automatically calculate boundary conditions for an imaged patient. Medial shape models called m-reps are used to facilitate these tasks and reduce the effort required to apply finite element analysis to image registration. Encouraging results are presented for the registration of CT image pairs which exhibit deformation caused by pressure from an endorectal imaging probe and deformation due to swelling.
\end{abstract}

Index Terms-registration, elastic, finite element, m-rep, prostate

\section{INTRODUCTION}

$\mathbf{F}$ INITE element analysis is a powerful computational tool for modeling soft tissue deformation. Most notably, this approach has yielded positive results for same-subject nonrigid image registration and for medical simulation applications. Published results include image registration for the prostate [1] [2] [3], brain [4] [5] [6] [7] [8], and breast [9] [10], as well as simulation of maxillo-facial surgery [11] and liver surgery [12] [13] [14]. In fact, finite element analysis produces such physically plausible biomechanical tissue deformations that the method is used to generate synthetic displacement fields that serve as a gold-standard in the validation of other deformable registration algorithms [15].

The algorithms presented here for automating finite element analysis are potentially applicable to a variety of medical imaging applications that require deformation modeling. We report results for the specific problem of prostate image registration, an important problem that arises during the planning and delivery of radiotherapy treatment for prostate cancer. Both external beam radiation and brachytherapy require registration between planning and treatment images. The need for deformable registration is particularly acute when magnetic resonance spectroscopy (MRS) images of the prostate are acquired using an endorectal imaging probe. MRS images are

Financial support for this work was provided by NIH grants CA P01 47982, EB P01 02779, and P01-CA59017. and by a Lucent Foundation GRPW fellowship.

J. Crouch is with the Computer Science Department at Old Dominion University.

S. Pizer and E. Chaney are with the Medical Image Display and Analysis group at the University of North Carolina, Chapel Hill.

Y. Hu, G. Mageras, and M. Zaider are with Memorial Sloan-Kettering Cancer Center valuable because they provide information about the location of tumor deposits within the prostate, but their use is complicated by the fact that pressure from an MRS imaging probe displaces the prostate upward and flattens it against the pubic bones. In order to use MRS images for treatment planning, a deformable registration between the planning and treatment images is required.

As computational power has grown more available, finite element analysis has become a more accessible tool for medical imaging research. However, its application is often limited to the research arena due to the substantial labor typically required to construct a high quality finite element model from a patient image. This work focuses on specific techniques to make finite element analysis more automatic and efficient for medical imaging applications. Reducing the labor required to generate a finite element model will make the method more attractive and practical for both research and clinical applications.

The remainder of this paper is organized as follows. Section II considers related prior work in prostate image registration. Section III provides an overview of the automated registration method, with sub-sections describing the methods used for shape modeling(III-B), finite element mesh generation (IIIC), boundary condition specification (III-D), and solution computation (III-E). The registration experiment is described in section IV, and the experiment results are presented in section V. Discussion of the results is contained in section VI, and section VII concludes the paper with a summary of planned future work.

\section{Previous Work}

Solutions to the problem of prostate registration have been proposed using both rigid [16] [17] and non-rigid [18] methods. Previous research has shown that the magnitude of prostate deformation is large enough that it should not be neglected in treatment planning, in particular for prostate brachytherapy [19]. Therefore we focus here only on deformable prostate registration methods. Different types of deformable prostate image registration approaches have been presented by [1], [2], and [3], each of which uses a finite element model as a component of the registration procedure.

The approach described by Bharatha, et al., [1] is most similar to the work presented here, and includes validation of finite element based prostate image registration using a linear elastic material model for magnetic resonance (MR) images. This earlier work differs from the method presentedIEEE 
here in several regards, including the algorithms for generating meshes and boundary conditions. In particular, meshes composed entirely of tetrahedra were used by Bharatha, et al., whereas the mesh algorithm employed in this work generates a hexahedral elements for the prostate. Hexahedral meshes provide numerical advantages over tetrahedral meshes in finite element analysis [20].

The prostate model presented by Alterovitz, et al. [2], uses a 2D finite element model and optimizes both the applied force vectors and the tissue stiffness parameters. As compared to the model developed in our work, the stiffness parameter optimization gives the Alterovitz model additional in-plane flexibility, but its limitation to two dimensions prevents it from representing any out-of-plane deformation.

Finally, the work presented by Mohamed, et al., [3] employs a combined statistical and biomechanical approach to prostate registration, while our work relies on medial geometry and biomechanics to generate a deformation. The consistent use of a medial model framework to automate the application of finite element analysis is a feature, described in the following sections, that sets our method apart from previous work.

\section{METHODS}

\section{A. Overview}

We present a multi-stage procedure for deformable image registration that involves image segmentation, mesh construction, boundary condition specification, solution of finite element equations, and displacement interpolation. Several of these stages rely on medially based shape models called mreps that provide a convenient representation of organ geometry along with an object based coordinate system, an essential feature for the automated registration procedure. The steps in the registration process are described below for a pair of 3D images, $I_{1}$ and $I_{2}$, that record a patient's prostate in different states of deformation. The inputs/outputs of each stage along with the dependencies between stages are depicted in Fig. 1.

1) Fit an m-rep model to the segmented prostate in image $I_{1}$, and label this instance of the m-rep $M_{1}$.

2) Fit the same m-rep model to the segmented prostate in image $I_{2}$, and label this instance of the m-rep $M_{2}$.

3) Build a $3 \mathrm{D}$ hexahedral finite element mesh for $M_{1}$.
4) Use the m-rep generated correspondences between points in $M_{1}$ and $M_{2}$ to estimate displacement vectors mapping the surface nodes of $M_{1}$ to corresponding points on the surface of $M_{2}$. Iteratively refine this estimate to get a set of essential finite element boundary conditions (displacements) that deform $M_{1}$ to match $M_{2}$ 's shape and simultaneously minimize the elastic energy of the solid object deformation.

5) Assemble the finite element system of equations using a linear elastic material model, the refined boundary conditions from step 4 , and the mesh from step 3. Solve this system to get a set of displacement vectors that map all the mesh nodes defined over the volume of $M_{1}$ onto $I_{2}$. Iteratively subdivide the mesh to arrive at a solution with the desired precision.

6) Interpolate the displacement field inside each 3D mesh element to determine the mapping from every voxel in $I_{1}$ to $I_{2}$

The following subsections describe the individual stages of the registration pipeline outlined above. Section III-B covers steps 1 and 2, discussing m-rep models and their use in image segmentation. Section III-C describes the hexahedral mesh generation algorithm used in step 3. Section III-D explains how finite element boundary conditions are formulated during step 4, and section III-E describes the solution method applied in step 5. The interpolation performed in step 6 is briefly discussed in section III-F.

\section{B. M-Rep Models}

M-rep models are used in this work to represent the 3D shape of an imaged prostate. Prior work has established algorithms for automatic image segmentation using m-reps and has provided data validating the success of those algorithms [21] [22]. A brief description of m-rep models and their use in segmentation is provided here.

Like all medially based shape representations, m-reps characterize the structure and geometry of an object using points on the object's medial surface. The simplest m-rep consists of a single figure that represents a slab-like region with a nonbranching medial locus. Because the prostate's shape can be

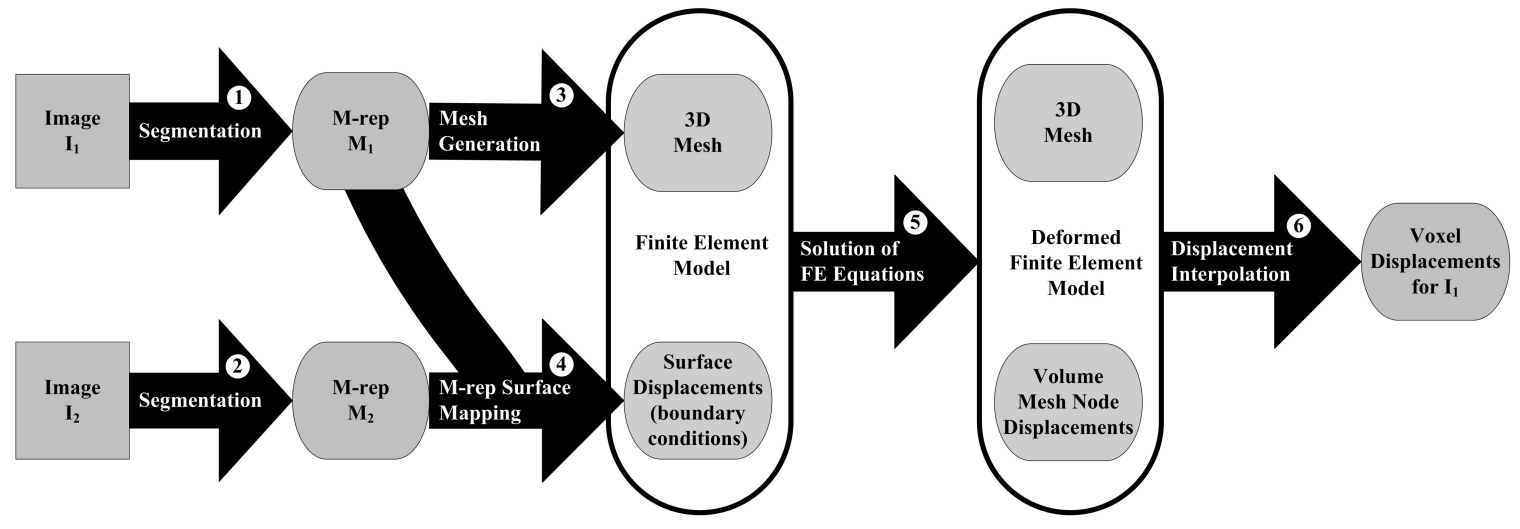

Fig. 1. These steps result in a deformation field that can be applied to Image $I_{1}$ to register it with Image $I_{2}$. 
well represented with a single figure m-rep, the discussion of m-reps here will be limited to single figure models.

A single m-rep figure is represented by a lattice of medial atoms, each of which provides a sample of object geometry at a point on the medial surface. Fig. 2 illustrates the structure of a medial atom and a figure. Each medial atom stores the coordinates of a point on the medial surface along with the object's radius around that point, two vectors originating at the medial point and terminating on the object's boundary ( $y_{0}$ and $y_{1}$ in Fig. 2), and other geometric information including an oriented coordinate frame. The lattice arrangement of medial atoms naturally leads to an object based coordinate system for m-reps. All points in an m-rep are referenced using $(u, v, t, \tau)$ coordinates, where $u$ and $v$ traverse the rows and columns of the atom lattice so that $1 \leq u \leq u_{\max }$ and $1 \leq v \leq v_{\max }$, where $u_{\max }$ equals the number of rows in the lattice and $v_{\max }$ equals the number of columns in the lattice. $\tau$ ranges from 0 at the medial surface to \pm 1 at the object boundary, and $t$ spans the angle between the two boundary vectors $y_{0}$ and $y_{1}$. The $t$ coordinate is important in the crest region of the object that is sampled by atoms on the edge of the medial surface; varying $t$ sweeps out the cap that joins the top $(\tau=+1)$ and bottom $(\tau=-1)$ portions of the object's boundary surface.

Given the discrete lattice of medial atom samples contained in an m-rep, a new medial atom can be computed at any $(u, v)$ point on the medial surface by smoothly interpolating the sample atoms' point coordinates, the object radius, and other attributes across the lattice. Thus for for any valid set of m-rep $(u, v, t, \tau)$ values, a corresponding $(x, y, z)$ world space point can be calculated. This mapping from medial coordinates to world coordinates is represented by the following function:

$$
\left[\begin{array}{lll}
x & y & z
\end{array}\right]=\text { ObjectToWorld }(\text { model }, u, v, t, \tau)
$$

In the implementation of Eqn. 1, different interpolation schemes are possible. In this work, surface interpolation is performed by defining bicubic Bézier spline patches over the medial surface and the boundary surface of the object. Interior point coordinates are mapped by using the $\tau$ coordinate to linearly interpolate between the $(x, y, z)$ coordinates of a point on the medial spline surface and a point on the boundary spline surface. An alternative interpolation method is described in [23].
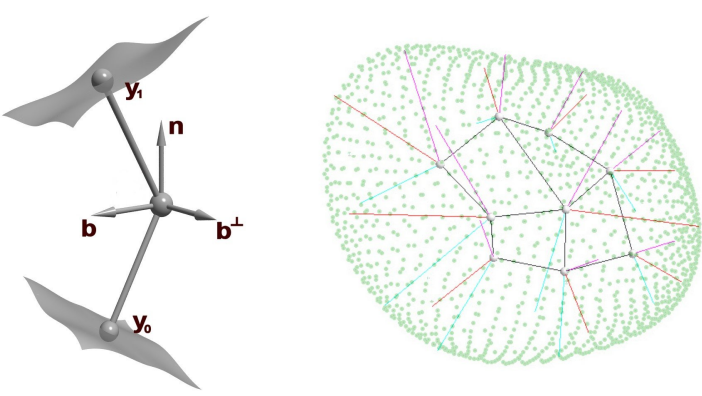

Fig. 2. Left: A medial atom with object properties labelled. $y_{0}$ and $y_{1}$ are vectors pointing to the object's boundary. $\left(n, b, b^{\perp}\right)$ defines the object's coordinate frame at the atom's location. Right: A single figure m-rep model composed of a lattice of medial atoms
M-rep models presented in this paper were created using a program named Pablo that was developed by the Medical Image Display and Analysis Group (MIDAG) group at the University of North Carolina at Chapel Hill. This software supports creation and editing of m-rep models and provides algorithms for automatically fitting an m-rep to grayscale image data via optimization [22]. Pablo fitted an m-rep model composed of a $3 \times 3$ lattice of atoms to segmented prostate images to produce the prostate shape models used in this work.

\section{Mesh Generation Algorithm}

Meshes composed of hexahedral elements are frequently preferable to meshes built from tetrahedral elements due to the superior convergence and accuracy characteristics of hexahedral elements [20]. A description of an automatic hexahedral element mesh generation algorithm based on m-reps follows in section III-C.1. Readers are referred to [24] and [25] for additional details on this meshing method. Section III-C.2 presents an algorithm for mesh optimization, and section IIIC.3 explains how mesh elements outside the prostate volume were created.

1) Hexahedral Mesh Generation: For single figure m-reps the automatic mesh generation algorithm creates a set of mesh nodes based on a standardized sampling pattern defined in terms of $(u, v, t, \tau)$ coordinates. The mapping defined in Eqn. 1 from object space coordinates to world space coordinates determines nodes' placement in world space. The standardized m-rep meshing pattern is illustrated in Fig. 3. The first step in meshing a m-rep figure is the construction of a sampling grid of mesh nodes on the $(u, v)$ parameter plane of the medial surface. The vertices of the sampling grid are placed at regular intervals in $(u, v)$ coordinates, as shown in Fig. 3b. Additional mesh nodes are then created by projecting the sampling grid on the medial surface out toward the object boundary. For all of the nodes on the medial surface except for those around the rim of the grid, this is accomplished by holding $u$ and $v$ constant and varying $\tau$. As shown in Fig. 3d, for a node on the interior of the medial surface grid five nodes are created at $\{\tau=-1.0,-0.5,0.0,0.5,1.0\}$. For sample points on the grid's rim, a set of six nodes is created, with the sixth node sitting on the object's crest. This pattern is illustrated in Fig. 3e; a slice through a fully meshed figure is shown in Fig. 3f.

Mesh construction is guided entirely by information contained in the m-rep model, so the meshing process described requires no user interaction. Note that a mesh defined in terms of medial object coordinates will fit any deformed version of the m-rep model that generated the mesh.

2) Mesh Optimization: Each three dimensional element is defined in a parameter space (Fig. 4a) and is mapped into world space (Fig. 4b) via the element shape function $(x, y, z)=N(\xi, \eta, \zeta)$. For the 8-node linear hexahedral elements used in this work, the shape function is defined as follows [26].

$$
N(\xi, \eta, \zeta)=\sum_{i=1}^{8} N_{i}(\xi, \eta, \zeta) \cdot\left[x_{i} y_{i} z_{i}\right]
$$




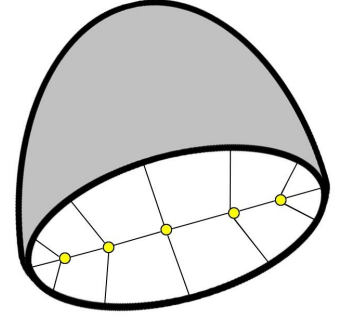

(a)

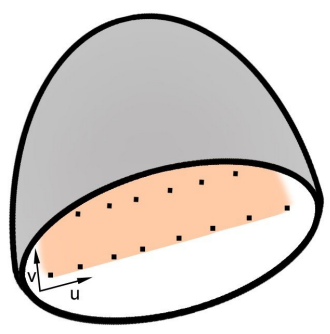

(c)

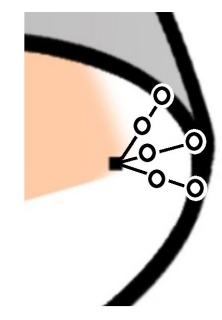

(e) (b)

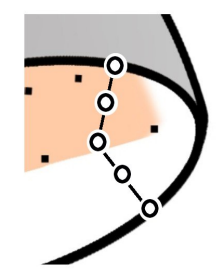

(d)

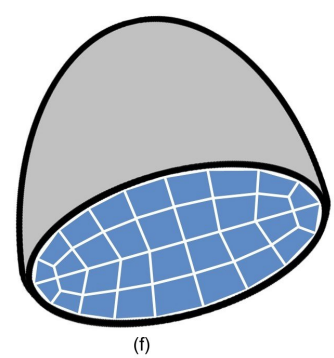

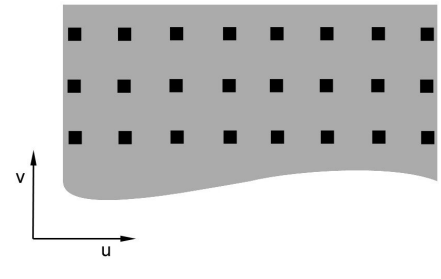

Fig. 3. (a) A slice through a single figure m-rep is shown, with one row of atoms visible. (b) A regular sampling grid of mesh nodes is defined in the $(\mathrm{u}, \mathrm{v})$ parameter space of the medial surface. (c) The regular sampling grid is mapped into world space. (d) Each sample point in the central portion of the medial surface gives rise to a group of five mesh nodes placed at constant $u$ and $v$ and evenly spaced $\tau$. Mesh nodes are drawn as black rings. (e) Sample points on the rim of the medial surface produce 6 mesh nodes. (f) A slice through a meshed figure shows the faces of hexahedral elements.

where $\left[\begin{array}{lll}x_{i} & y_{i} & z_{i}\end{array}\right]$ are the coordinates of node $i$ and

$$
N_{i}=\frac{1}{8}(1 \pm \xi)(1 \pm \eta)(1 \pm \zeta)
$$

The existance of a valid finite element solution for a given mesh element depends on $J$, the Jacobian of the element's shape function $N(\xi, \eta, \zeta)$. Although a deformation solution can be computed as long as the measure of element volume, $\operatorname{det}(\mathrm{J})$, is non-negative [27], when $\operatorname{det}(\mathrm{J})$ is scaled to the interval $[-1,1]$ by normalizing for element size, desirable elements have a value of at least 0.5 . Smaller $\operatorname{det}(\mathrm{J})$ values are associated with strongly distorted elements that can adversely impact the condition number of the finite element stiffness matrix and reduce the accuracy of the computed solution. It is sufficient to check the $\operatorname{det}(\mathrm{J})$ values at the Gauss integration points in each element [28]. Computing the stiffness matrix requires numerical evaluation of an integral over the volume of the entire mesh; the Gauss integration points are the locations inside each element where this integral is evaluated.

If elements with low $\operatorname{det}(\mathrm{J})$ values are generated, they can be improved through optimization. The mesh quality improvement procedure first assigns each element a score that is the minimum value of $\operatorname{det}(\mathrm{J})$ evaluated at each of eight Gauss integration points in the element. A list is compiled of all elements with $\operatorname{det}(\mathbf{J})$ values less than 0.5 and the immediate neighbors of those elements. For each element on the list, the coordinates of its nodes are varied using a Nelder-Mead simplex search [29] optimization procedure that maximizes the element's $\operatorname{det}(\mathbf{J})$ value. A prostate mesh produced through this process is shown in Fig. 5.

In order to make the mesh optimization process robust and efficient we use two new m-rep parameterizations that are defined specifically for use in optimization. The first is an invertible mapping between any valid $(u, v, t, \tau)$ coordinates and the three dimensional $(A, B, C)$ coordinate space. Over the central portion of a figure, $(A, B, C)$ coordinates are defined by mapping $u \rightarrow A, v \rightarrow B$, and $\tau \rightarrow C$. In the crest region of an object trigonometric formulas are applied to map the region spanned by the medial coordinate, $t$, into $(A, B, C)$. Details of this mapping are provided in the appendix.

The second coordinate space employed is two dimensional and spans the surface of an m-rep figure. Optimizing the position of surface nodes in this coordinate space implicitly constrains the nodes to remain on the object's surface. Intuitively, $(a, b)$ coordinates are computed by unwrapping the surface of a figure's $(A, B, C)$ coordinate space and projecting it onto a plane. Details are again provided in the appendix.

3) External Region Meshes: It is desirable to mesh a region outside the modeled object so that a smooth deformation can be applied to the surrounding tissue. The m-rep meshing algorithm does not address the problem of meshing non-modeled areas. Therefore for the prostate registration experiments a tetrahedral mesh is generated to fill the volume of interest surrounding the prostate. A layer of pyramid elements provides an interface between the hexahedral and tetrahedral elements. The base of a pyramid element is placed against the exposed quadrilateral face of each of the outermost prostate hexahedral elements. All the exposed pyramid element faces are then triangles, and a tetrahedral mesh fills in gaps between these triangles and the boundary of the region of interest. The tetrahedral meshes used in this work are generated by CUBIT, a meshing program from Sandia National Laboratories. The pyramids and tetrahedra that fill the space external to the $\mathrm{m}$ rep models are shown in Figs. 5d and 5e.
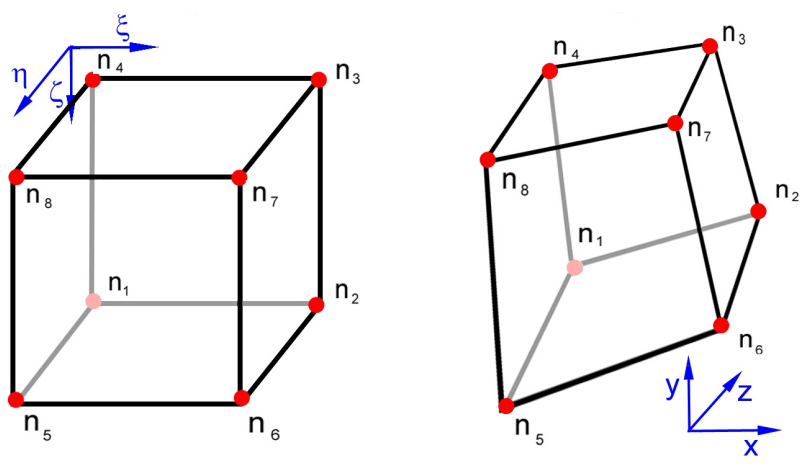

Fig. 4. Left: Hexahedral element in its $(\xi, \eta, \zeta)$ parameter space Right: Hexahedral element that has been mapped into world space 


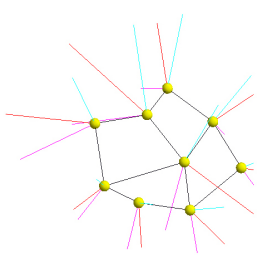

(a)

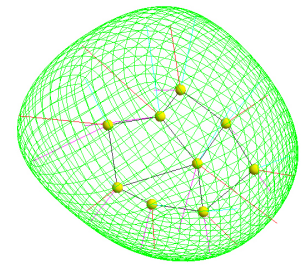

(b)

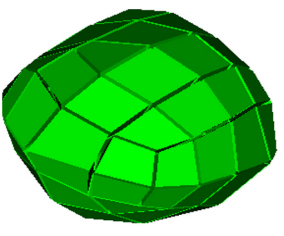

(c)
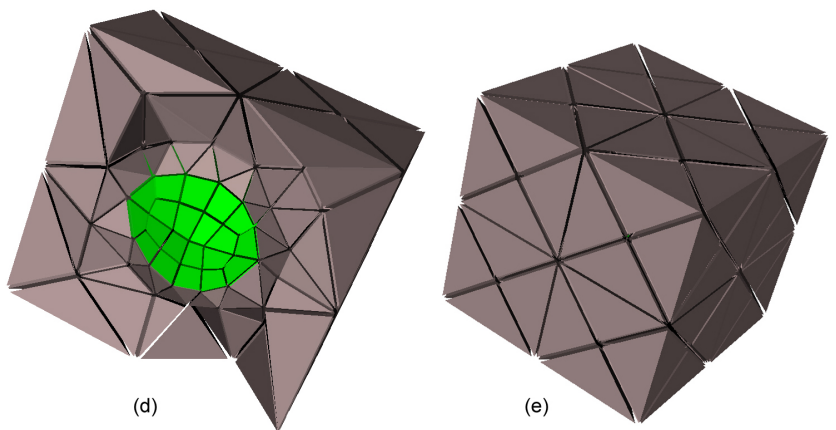

Fig. 5. (a) M-rep model of a prostate (b) Prostate m-rep with implied surface (c) Base level prostate mesh (d) Sliced view of the meshed volume of interest (e) Exterior view of the meshed volume of interest

\section{Boundary Conditions}

To compute a deformation with finite element analysis, boundary conditions are specified for some of the nodes in terms of node displacements. However, point displacements are not available directly from the images. What is visible in the images is shifting and/or change in the shape of the object boundary. Thus we use the m-rep model to derive an initial approximation to surface point displacements from observed boundary changes.

In the case of the prostate, the m-rep model $M_{1}$ is fitted to image $I_{1}$ and is used to guide mesh construction. Then $M_{1}$ is transferred onto the image of the deformed prostate where it is fitted to the deformed prostate shape, yielding $M_{2} . M_{1}$ and $M_{2}$ have the same topology, and their object coordinates span exactly the same parameter space. This means that the mreps' object coordinates implicitly define a one to one mapping between points in the original prostate and points in the deformed prostate. For a node belonging to $M_{1}$ with medial coordinates $\left(u_{i}, v_{i}, t_{i}, \tau_{i}\right)$, an approximate displacement vector $d_{i}$ is defined in the following way.

$$
\begin{aligned}
& {\left[\begin{array}{lll}
x_{(i, 1)} & y_{(i, 1)} & z_{(i, 1)}
\end{array}\right]=\operatorname{ObjectToWorld}\left(M_{1}, u_{i}, v_{i}, t_{i}, \tau_{i}\right)} \\
& {\left[\begin{array}{lll}
x_{(i, 2)} & y_{(i, 2)} & z_{(i, 2)}
\end{array}\right]=\operatorname{ObjectToWorld}\left(M_{2}, u_{i}, v_{i}, t_{i}, \tau_{i}\right)} \\
& d_{i}=\left[\begin{array}{lll}
x_{(i, 2)} & y_{(i, 2)} & z_{(i, 2)}
\end{array}\right]-\left[\begin{array}{lll}
x_{(i, 1)} & y_{(i, 1)} & z_{(i, 1)}
\end{array}\right]
\end{aligned}
$$

A full set of boundary conditions for a prostate model consists of the vectors $d_{i}$ that specify displacements for nodes on the prostate surface and zero displacement vectors for nodes on the exterior surface of the whole meshed volume, specifying that those nodes remain stationary. The exterior surface nodes are the ones lying on the outermost faces of the tetrahedra seen in Fig. 5e.

Applying this set of boundary conditions to the finite element mesh will result in a deformed object that exhibits the shape observed in image $I_{2}$. This geometry based approximation of the boundary conditions is not unique; any non-folding surface-to-surface mapping between m-rep models $M_{1}$ and $M_{2}$ will provide displacement boundary conditions that produce the required shape change. To increase the accuracy of the computed deformation, the set of boundary conditions can be optimized so that the energy of the deformation is minimized. Boundary condition optimization relies on the assumption that when multiple sets of boundary conditions all result in the same boundary shape change, the most likely set is the one that requires the least amount of energy to produce the deformation.

Since non-zero boundary conditions are only applied to nodes on the prostate's surface, boundary condition optimization is most efficiently performed using the nodes' $(a, b)$ surface coordinates. For the $i^{t h}$ surface node, $\left(a_{(i, 1)}, b_{(i, 1)}\right)$ references a point on the surface of the original m-rep model $M_{1}$ and $\left(a_{(i, 2)}, b_{(i, 2)}\right)$ denotes a point on the surface of the deformed model $M_{2}$. The displacement $d_{i}$ can then be defined as follows.

$$
\begin{aligned}
& {\left[\begin{array}{lll}
x_{(i, 1)} & y_{(i, 1)} & z_{(i, 1)}
\end{array}\right]=\operatorname{SurfaceToWorld}\left(M_{1}, a_{(i, 1)}, b_{(i, 1)}\right)} \\
& {\left[\begin{array}{lll}
x_{(i, 2)} & y_{(i, 2)} & z_{(i, 2)}
\end{array}\right]=\operatorname{SurfaceToWorld}\left(M_{2}, a_{(i, 2)}, b_{(i, 2)}\right)} \\
& \\
& d_{i}=\left[\begin{array}{lll}
x_{(i, 2)} & y_{(i, 2)} & z_{(i, 2)}
\end{array}\right]-\left[\begin{array}{lll}
x_{(i, 1)} & y_{(i, 1)} & z_{(i, 1)}
\end{array}\right]
\end{aligned}
$$

The SurfaceToWorld transform maps the $(a, b)$ coordinates to medial $(u, v, t, \tau)$ coordinates using the relations detailed in the appendix, and then applies the ObjectToWorld transform to the $(u, v, t, \tau)$ coordinates.

The boundary condition optimization procedure initializes the surface point correspondences by setting $\left(a_{(i, 2)}, b_{(i, 2)}\right)=$ $\left(a_{(i, 1)}, b_{(i, 1)}\right)$. Then the $\left(a_{(i, 2)}, b_{(i, 2)}\right)$ coordinates are adjusted by a Nelder-Mead simplex search algorithm [29] to minimize the strain energy of the resulting deformation. This optimization is illustrated in Fig. 6. The strain energy is defined as

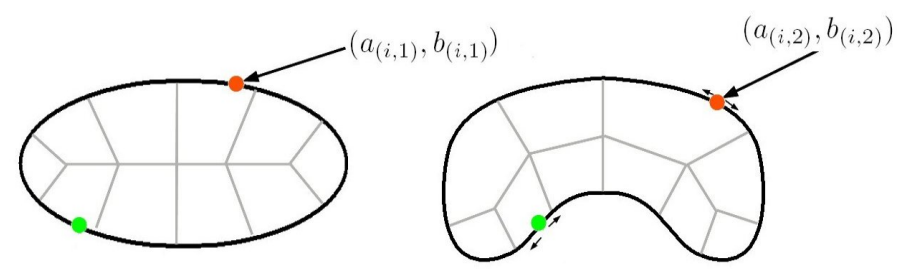

Fig. 6. In the boundary condition optimization process, $(u, v, t, \tau)$ coordinates are converted to the more compact $(a, b)$ surface coordinates. Corresponding points are then allowed to slide along the object surface, as shown on the right, to minimize the energy of a deformation. 
follows [30] [31].

$$
P E=\frac{1}{2} \int_{V} \sigma \cdot \varepsilon \mathrm{d} V \quad \text { where } \quad \begin{aligned}
\sigma & \text { is the stress } \\
\varepsilon & \text { is the strain } \\
V & \text { is the object volume }
\end{aligned}
$$

\section{E. Solution Algorithm}

To compute the deformation of a three dimensional linear elastic finite element model with $\mathrm{N}$ nodes, a system of linear equations of the following form must be solved.

$$
K a=f
$$

where $\quad K \quad$ is the $3 \mathrm{~N} \times 3 \mathrm{~N}$ stiffness matrix

$a$ is a vector containing node displacements

$f \quad$ is a vector containing node forces

An initial approximation to the deformation solution can be derived from the m-rep model correspondences in the same way that the initial approximation to the boundary conditions is derived. For each node in the mesh, an initial approximation to the node's displacement vector $d_{i}$ is defined by Eqn. 3. A preconditioned conjugate gradient solver is used to iteratively improve the solution to Eqn. 6.

1) Mesh Subdivision: If greater accuracy than that provided by the initial mesh is desired, the mesh can be subdivided to create smaller elements that provide a higher resolution deformation solution. The mesh nodes produced by the $\mathrm{m}$ rep meshing algorithm have both world space $(x, y, z)$, and medial object $(u, v, t, \tau)$ coordinates. By subdividing elements using the object coordinate system, a smoother, more detailed approximation of the object geometry is produced. Straightforward subdivision with world space coordinates would provide higher resolution for the solution representation but would not change the error of the geometric representation. The medial coordinate based subdivision process allows for increased precision in both the geometry and the solution. The advantage of increased geometric precision is a reduction in deformation error in the vicinity of the boundary. Subdivision using world space coordinates is compared to subdivision using medial object coordinates in Fig. 7. The result of applying medial coordinate based subdivision to a prostate mesh is shown in Fig. 8. Each subdivision step increases the size of the system of equations by approximately a factor of 8 .

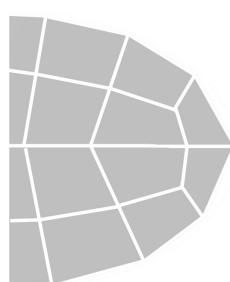

(a)

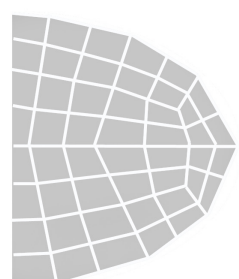

(b)

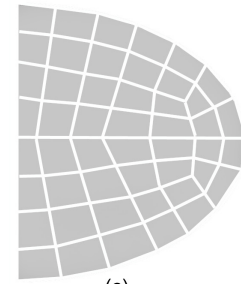

(c)
Fig. 7. (a) Coarse mesh. (b) Subdivision of coarse mesh using Euclidean coordinate midpoint computations. (c) Subdivision of coarse mesh using medial coordinate midpoint computations. The subdivision in (c) is a smoother approximation of the object's boundary than the subdivision in (b).
2) Solution on a Subdivided Mesh: The larger system of finite element equations that results from subdivision can be efficiently solved by taking a multiscale approach and using sparse matrix data structures. The approach taken here is to solve the finite element system of equations on the initial mesh using the procedure outlined at the beginning of section III-E and then interpolate that solution to the subdivided mesh and solve again using a conjugate gradient solver.

\section{F. Displacement Interpolation}

The solution to Eqn. 6 provides a 3D displacement vector for each mesh node. To find the displacement vector for a voxel in $I_{1}$, a spatial search is performed on the mesh to determine the element index and $(\xi, \eta, \zeta)$ coordinates that correspond to the voxel's location. Then by substituting the node displacement vectors for the node coordinates in Eqn. 2, the shape function $N(\xi, \eta, \zeta)$ is used to interpolate the displacement to the voxel's location. The interpolated displacement vector maps the voxel from $I_{1}$ onto the corresponding point in image $I_{2}$.

\section{REgistration EXPERIMENT}

Validation of the finite element registration method was accomplished through prostate image registration experiments. In these experiments a single figure m-rep model represented the prostate. Pablo fitted the m-rep to binary prostate images that were produced from expert manual segmentations of CT image pairs. The area around the prostate was represented as a homogeneous region, and all soft tissues were modeled as isotropic linear elastic solids. Boundary conditions were supplied in terms of displacement vectors for nodes on the prostate model's surface. The material parameters for the linear elastic material model were $E$, Young's modulus, and $\nu$, Poisson's ratio. Since boundary conditions were specified in terms of displacement vectors rather than force vectors, the deformation did not depend on Young's modulus [25]. Poisson's ratio was set to $\nu=0.49$ for the prostate, indicating near incompressibility.

To be meaningful, the method for evaluating the registration accuracy needed to be independent of the method used to produce the registrations. Since expert prostate surface segmentations were an experiment input, the registration

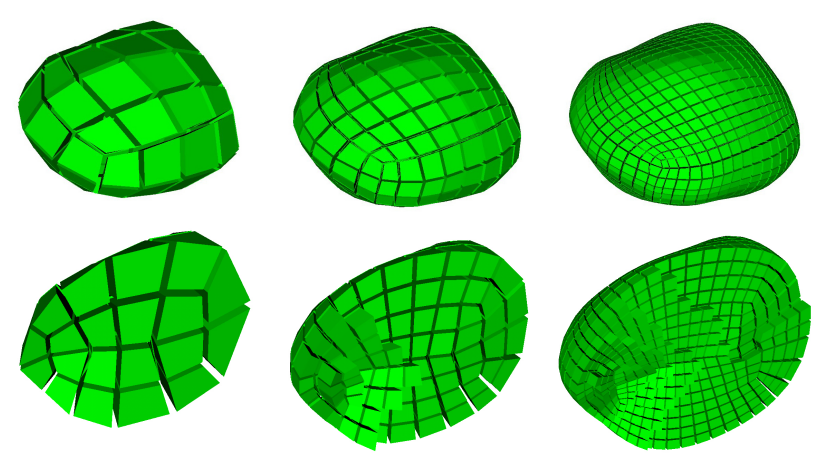

Fig. 8. Top row: Surface of a prostate mesh at subdivision levels $1-3$ Bottom row: Sliced view of a prostate mesh at subdivision levels 1 - 3 
accuracy could not be evaluated in terms of percentage of prostate volume overlap, prostate surface proximity, or any other metric derived from an analysis of registered prostate surface positions. Instead, it was appropriate to evaluate these registrations by examining the alignment of corresponding fiducial points inside the registered prostate volume. Since fiducial points were not used at any stage of the finite element registration process, they provided a truly independent measure of registration accuracy. Implanted brachytherapy seeds were the fiducials employed in the validation experiment. Seed voxels were automatically located in the test images via image intensity thresholding, and seed coordinates were computed as the average position of a cluster of bright voxels. A mapping between the seeds extracted from each of two images was established through the use of a non-rigid point matching algorithm [32].

The validation study was performed using computed tomography (CT) prostate images acquired at Memorial SloanKettering Cancer Center. Results are presented for the following pairs of prostate $\mathrm{CT}$ images:

$\mathcal{A})$ Prostate phantom deformed by MR imaging probe A deformable male pelvis phantom was implanted with non-radioactive brachytherapy seeds and scanned first with a deflated MR imaging probe in the rectum (image $\mathcal{A}_{1}$ ) and then scanned again with the probe inflated (image $\mathcal{A}_{2}$ ). This produced a pair of images of the prostate and surrounding region that captured the displacement and deformation caused by pressure from the probe. The phantom's prostate, bladder, and rectum were made of a water-based polymer designed to simulate the properties of real tissues (Computerized Imaging Reference Systems, Inc.). 75 brachytherapy seeds were evenly distributed in the prostate and visible in both images. The in-plane resolution for this volumetric pair of images was $0.49 \mathrm{~mm}$, and the thickness of each slice was $1.5 \mathrm{~mm}$. A representative slice from each of the CT images in this pair is shown in Fig. 9.

$\mathcal{B})$ Patient prostate deformed by swelling

A brachytherapy patient was scanned 1 hour after 68 seeds were implanted (image $\mathcal{B}_{1}$ ) and again one month later (image $\mathcal{B}_{2}$ ). In the first scan the prostate was enlarged due to swelling caused by the brachytherapy procedure. The in-plane resolution for this pair of images
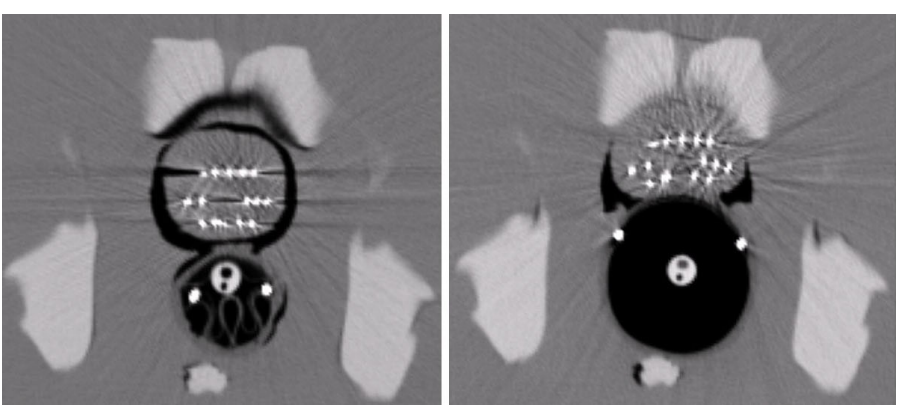

Fig. 9. Left: A CT slice from image $\mathcal{A}_{1}$ shows the phantom prostate with the uninflated probe. Right: A CT slice from image $\mathcal{A}_{2}$ shows the phantom prostate with the inflated probe. was $0.49 \mathrm{~mm}$, and the thickness of each slice was 5.0 $\mathrm{mm}$.

For comparison purposes, a fluid based registration method was also applied to the prostate image pairs [33]. This method produced a registration by optimizing the deformation field to maximize image intensity match. Because brachytherapy seeds show up brightly and distinctly in CT images, the presence of the seeds strongly affected the result of this intensity driven registration algorithm. To remove this bias and permit the fluid registration to be legitimately evaluated using the same metric as the finite element registration method, the brachytherapy seeds were edited out of the prostate images and the fluid method was applied to the edited CT images. The editing was accomplished through the following steps:

1. All voxels inside the segmented prostate volume were classified as seed voxels or prostate tissue voxels via intensity thresholding.

2. The mean and standard deviation of the intensity of prostate tissue voxels was computed.

3. The intensity of each seed voxel was replaced by a randomly selected intensity from the distribution computed in the previous step.

Since the prostate tissue in the test images appeared homogeneous on CT except for the presence of the seeds, the edited images visually matched the expectation for prostate images without seeds. An edited patient image slice is shown in Fig. 10.

\section{REsults}

This section first presents registration accuracy results and then analyzes algorithm performance. Registration accuracy is reported based on measurements of brachytherapy seed alignment between registered prostate image pairs. The discussion of algorithm performance considers the computational efficiency of the solution method and the effectiveness of the mesh and boundary condition optimization procedures.

\section{A. Registration Accuracy}

The registration accuracy is clinically most significant in the anterior/posterior direction since the prostate is adjacent to the anterior wall of the rectum. Accurate registration in this direction is necessary to minimize the rectum's exposure

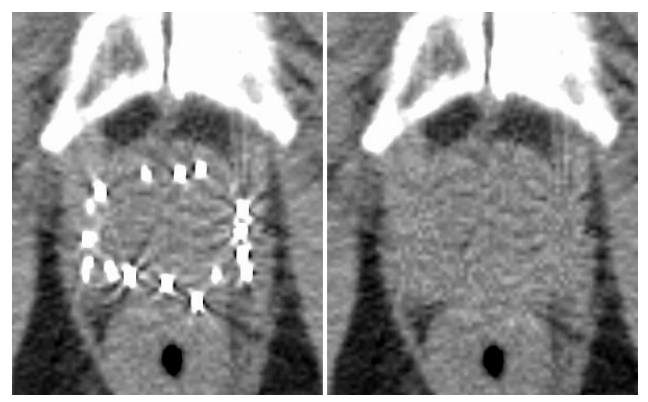

Fig. 10. Left: A portion of an axial slice from a patient prostate CT image is shown. Right: The same CT slice is shown with the brachytherapy seeds edited out of the image via the procedure described in section IV. 
to radiation which can cause complications including rectal bleeding. When prostate deformation is caused by pressure from an endorectal imaging probe, as in image pair $\mathcal{A}$, the deformation is greatest in the anterior/posterior direction. Therefore in addition to calculating the total registration error, the magnitude of the error component in each of the left/right, anterior/posterior, and superior/inferior directions was assessed.

For both test image pairs, the finite element registration error was of the same order as the image resolution. This is the best registration accuracy that could be expected since uncertainty in seed segmentations contributed to the registration error measurements, and the image resolution was the limiting factor for seed segmentation accuracy. In both test cases, the fluid registration method produced errors that were greater than the finite element registration errors and significantly larger than the image resolution.

The registration error at each seed was computed as the difference between the seed's registered image $\mathcal{A}_{1}$ or $\mathcal{B}_{1}$ coordinates and its corresponding image $\mathcal{A}_{2}$ or $\mathcal{B}_{2}$ coordinates. For each of the test image pairs the mean and standard deviation of the seed registration error are reported in Figs. 11 and 12 for the set of all seeds that were identified with high confidence in both of the paired images. The point matching algorithm successfully matched more than $75 \%$ of the seeds in each of the image pairs; 58 of the 75 seeds implanted in the phantom were matched and 52 of the 68 seeds implanted in the patient were matched. The seeds that could not be matched with confidence by the point matching algorithm were excluded from the error analysis. However, the regular nature

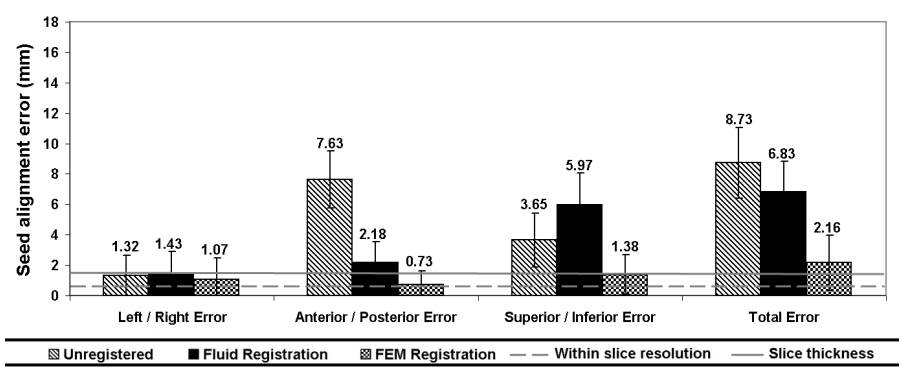

Fig. 11. Average registration error and standard deviation are shown for brachytherapy seeds embedded in a phantom prostate (image pair $\mathcal{A}$ ). The image resolution in the left/right and anterior/posterior directions is $0.49 \mathrm{~mm}$, indicated in the graph with a dashed line. The slice thickness in the superior/inferior direction is $1.5 \mathrm{~mm}$, indicated with a solid gray line.

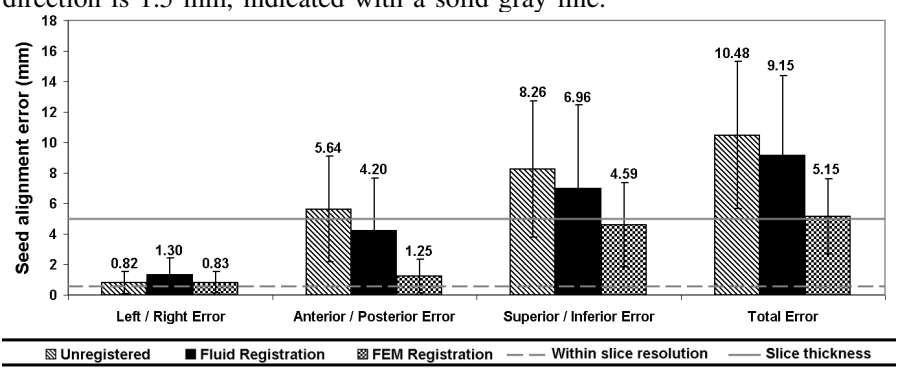

Fig. 12. Average registration error and standard deviation are shown for brachytherapy seeds embedded in a patient's prostate (image pair $\mathcal{B}$ ). The image resolution in the left/right and anterior/posterior directions is $0.49 \mathrm{~mm}$. The slice thickness in the superior/inferior direction is $5.0 \mathrm{~mm}$. of the seed distribution in the phantom allowed all 75 of the phantom seeds to be manually matched between images $\mathcal{A}_{1}$ and $\mathcal{A}_{2}$. The manual and automatic seed point matchings were consistent, and there was no significant difference between the error metrics computed for the 75 manually matched seeds and the 58 automatically matched seeds. This indicates that the error metrics computed for the partial seed matchings are reliable.

1) Registration accuracy for phantom images: The average amount of seed movement observed in image pair $\mathcal{A}$ due to the inflation of the MR imaging probe was $10.48 \mathrm{~mm}$. After finite element registration the average alignment error in the anterior/posterior direction was $1.07 \mathrm{~mm}$. Fig. 11 summarizes the registration error for the set of all matched seeds when registered by the finite element method and when registered by the fluid method. Note that the fluid registration error is significantly larger than the finite element error in the superior/inferior direction and in the most important anterior/posterior direction.

Fig. 13 illustrates the structure of the deformation field computed for image pair $\mathcal{A}$ by applying the deformation to a slice of a grid image and to the slice of image $\mathcal{A}_{1}$ shown undeformed in the left panel of Fig. 9. The positions of the brachytherapy seeds in this computationally deformed image closely match their positions in the image $\mathcal{A}_{2}$, previously shown in the right panel of Fig. 9.

When the m-rep geometry-based point correspondences defined in Eqn. 3 were used to register image pair $\mathcal{A}$, the registration error averaged $3 \mathrm{~mm}$ per seed with $2 \mathrm{~mm}$ of the error in the important anterior/posterior direction. Thus computing the deformation field using the finite element model eliminated more than half of the anterior/posterior registration error found in the geometry-based mapping.

2) Registration accuracy for patient images: Image pair $\mathcal{B}$ exhibits prostate deformation due to tissue swelling and includes the influence of real tissue inhomogenity. In this regard, test image pair $\mathcal{B}$ provides an excellent test for the registration algorithm. The larger slice thickness used in the clinical images limits the precision of error measurement in the superior/inferior direction. However the in-slice resolution of this image pair is the same as that of the phantom images and permits careful examination of the anterior/posterior error component, which averaged $1.25 \mathrm{~mm}$ per seed. In contrast, the fluid registration error in the same direction averaged 4.20 $\mathrm{mm}$ per seed. Fig. 14 shows slices from image $\mathcal{B}_{2}$ along with registered seeds segmented from images $\mathcal{B}_{1}$ and $\mathcal{B}_{2}$.

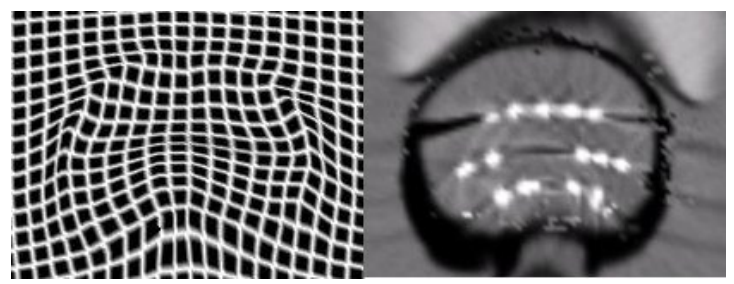

Fig. 13. Left: The computed deformation for the registration of image pair $\mathcal{A}$ applied to a regular grid. Right: The same deformation applied to a slice of the CT with the uninflated probe. 

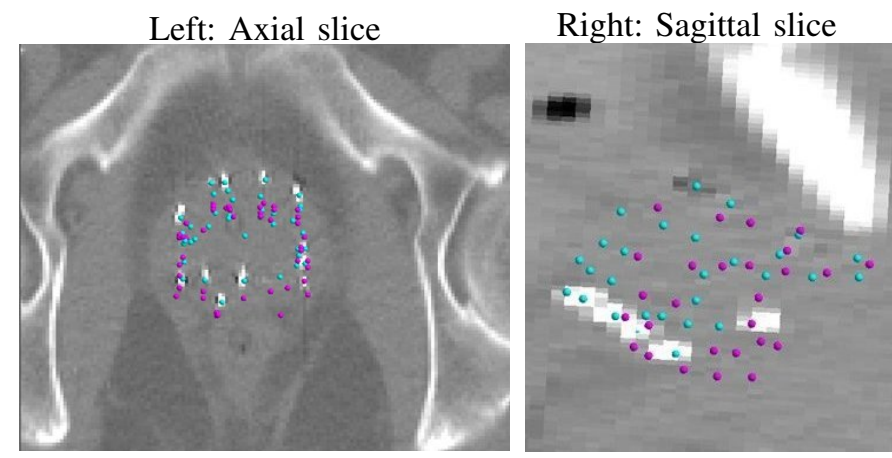

Top row: Unregistered seeds
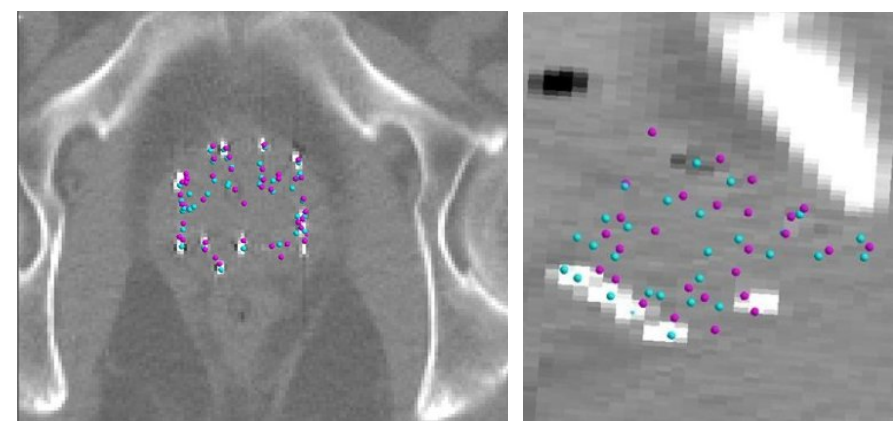

Center row: Seeds registered via finite element method
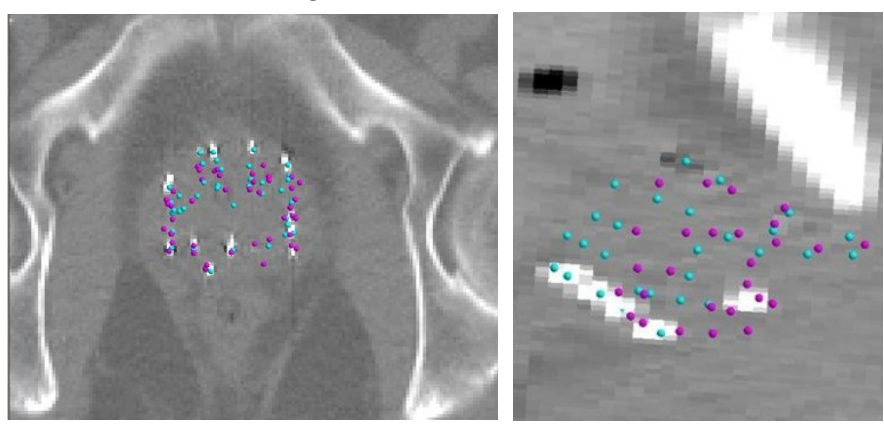

Bottom row: Seeds registered via fluid method

Fig. 14. Axial and sagittal slices of image $\mathcal{B}_{2}$ are shown. Seed positions segmented from $\mathcal{B}_{2}$ are rendered in cyan. The magenta seeds were identified in image $\mathcal{B}_{1}$ and mapped onto $\mathcal{B}_{2}$ with no registration (top), finite element registration (center) and fluid registration (bottom). In both the axial and sagittal views, some seeds are not visible because they are located behind the image slice.

\section{B. Algorithm Performance}

Performance measures for the finite element mesh optimization and boundary condition optimization algorithms are reported here, followed by a discussion of the computational efficiency of the solution algorithm.

1) Mesh Quality Optimization: The majority of prostate mesh elements created by the meshing algorithm presented in section III-C were of high quality and $\operatorname{had} \operatorname{det}(\mathrm{J})>0.5$ prior to any optimization. Fig. 15(a) displays a histogram of $\operatorname{det}(\mathrm{J})$ for an initial prostate phantom mesh. The optimization process described in section III-C.2 succeeded in improving the hexahedral prostate meshes so that $\operatorname{det}(\mathrm{J})>0.5$ for all elements, as shown in Fig. 15(b). The element shape quality of the prostate meshes remained high after each of three subdivision steps, making repeated application of the mesh optimization step unnecessary for the prostate models.
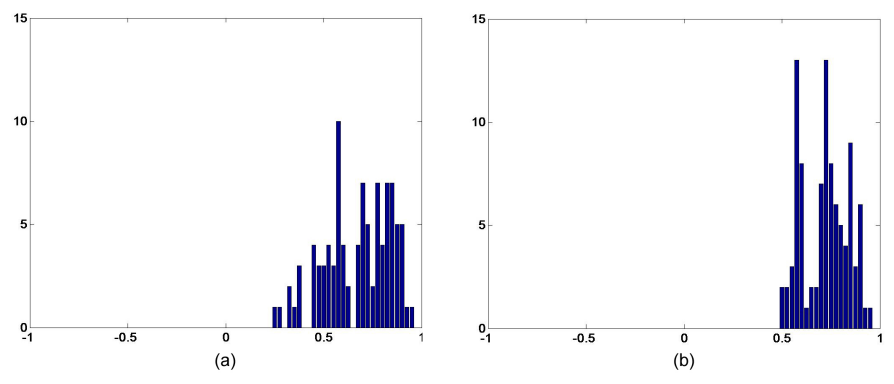

Fig. 15. Histograms of $\operatorname{det}(\mathrm{J})$ for elements of the phantom prostate mesh (a) before element quality optimization and (b) after element quality optimization

2) Boundary Condition Optimization: Tests showed that the optimization procedure described in section III-D reduced the energy of a prostate deformation by up to $20 \%$, but the optimization had a negligible impact on the registration accuracy at the seeds. The displacement vectors for the seed points changed by such small amounts after optimization that the error statistics were unaffected.

3) Computational Efficiency: The experiment results demonstrated that the number of solver iterations required to converge to a solution was reduced due to the good initial approximation provided by a solution on a coarser mesh. Fig. 16 shows the number of conjugate gradient iterations required for solution convergence after three mesh subdivision steps and shows that the computational savings gained by starting with the solution predicted from a coarser level increases with the subdivision level.

The finite element code used for this experiment was implemented in Matlab, leading to slower execution times than a compiled and optimized implementation of the algorithms presented would provide. With the Matlab implementation a deformation of prostate mesh level 1 or 2 could be computed on a desktop workstation in several minutes. A solution on mesh level 3 required about 12 hours. Timing runs with the authors' more recently developed finite element code that is written in $\mathrm{C}++$ indicates that if the prostate experiment were repeated using the new code the solution for the level 1 mesh would be computed in less than 0.1 seconds, the solution for the level 2 mesh would be computed in less than 2 seconds, and the solution for the level 3 mesh would be computed in less than 2 minutes.

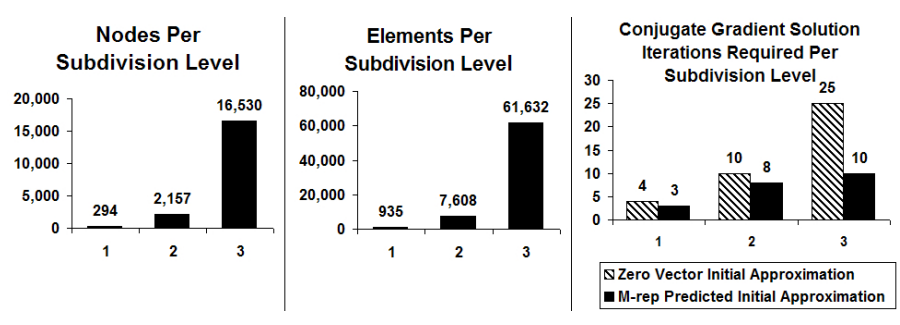

Fig. 16. The number of nodes, elements, and solution iterations required for prostate mesh subdivision levels 1 - 3 using image pair $\mathcal{A}$. The rightmost graph compares the solution iteration counts required using a zero displacement initial solution approximation compared to the iteration counts required using $\mathrm{m}$-rep predicted displacements as the solution approximation. 


\section{DISCUSSION}

An analysis of the registration experiment results reported in the previous section is provided below. The registration error measurements for the finite element and fluid methods are considered first, followed by a discussion of the performance of the finite element registration algorithm.

\section{A. Registration Accuracy}

The clinical goal for prostate image registration is $2 \mathrm{~mm}$ accuracy, with the greatest importance given to the error component in the anterior/posterior direction. The finite element registration results are encouraging because they indicate the $2 \mathrm{~mm}$ goal can be achieved when accurately segmented high resolution images are provided; even with thick $5.0 \mathrm{~mm}$ image slices the registration in the anterior/posterior direction is excellent, averaging $1.25 \mathrm{~mm}$ error for the patient test case.

The larger errors produced by the fluid method in the registration experiment can be attributed to the lack of visible structure inside the prostate in CT images. Even the prostate's boundary was difficult to detect in some regions, putting any intensity driven registration approach at a disadvantage. The finite element method did not suffer from this disadvantage because the input segmentations and the m-rep models provided a continuous prostate surface even where the boundary was not clearly visible in the images. Thus the prostate shape information encapsulated in the models allowed the finite element method to generate more accurate prostate deformations than the fluid method.

\section{B. Algorithm Performance}

The significance of the finite element registration algorithm is considered here, with special attention given to the novel aspects of the mesh generation and optimization procedures.

As previously noted, hexahedral finite element meshes can often provide better convergence and accuracy than the more commonly used tetrahedral meshes. Research has shown that for both linear elastic and non-linear elasto-plastic problems the error in a finite element solution is smaller when a mesh of linear hexahedral elements is used than when a mesh of similarly sized linear tetrahedral elements is used [20]. Research has been directed toward the development of hexahedral meshes from images [34], but the development of automatic hexahedral meshing algorithms is a challenging problem that continues to motivate current research efforts in the mesh generation community [35]. Therefore, the automatic generation of quality hexahedral meshes by the m-rep based meshing algorithm is a noteworthy development.

As detailed in section V-A.1, the element shape optimization succeeded in improving element quality and the boundary condition optimization succeeded in discovering sets of boundary conditions that resulted in lower energy deformations. However, neither of these optimizations were critical to the outcome of the prostate registration experiment, and when the tests were repeated without the optimizations, the registration error was not significantly different. The availability of these optimization procedures could still be important for situations where the deforming structures have more complex geometry than the prostate, since the initial m-rep approximations may be less accurate in such cases.

From a computational point of view, both types of optimization were made more efficient and robust through the introduction of alternate parameterizations of the m-rep model volume and surface. The mapping from $(u, v, t, \tau)$ medial coordinates to the three dimensional $(A, B, C)$ volume coordinates and the two dimensional $(a, b)$ surface coordinates improved optimization efficiency because the point representations provided by these parameterizations was more compact and reduced the dimensionality of the space searched during the optimizations.

\section{CONCLUSION \& Future WORK}

We conclude that the finite element image registration algorithm presented here shows promise for producing deformable registrations of $3 \mathrm{D}$ prostate images with a minimal amount of human intervention. The accuracy levels achieved with the method seem to be primarily limited by the segmentation accuracy and input image resolution.

In addition to software engineering efforts, future work will proceed in three directions. First, the prostate registration algorithm may be expanded to use a five object male pelvis model that includes the rectum, bladder, and pubic bone objects in addition to the prostate. The use of a multi-object model would allow not only the prostate but also the surrounding structures to be accurately registered. Second, the registration algorithm will be applied to images of other soft tissue organs such as the liver. Recent methods of radiation treatment such as high dose treatment and tomotherapy require tight treatment margins. Such tight margins can be achieved through highly accurate registration of planning and treatment images. Finally, more advanced material modeling will be pursued. Spatially varying material parameters could increase the realism of the model, as could the addition of more complex material models. Soft tissues are most accurately characterized as viscoelastic [36], so it is important to determine whether registration accuracy might be improved through the use of a viscoelastic or hyperelastic constitutive model.

\section{APPENDIX I \\ Medial ObJect CoORdinate Transforms}

The coordinate transformations used in the mesh element optimization (section III.C) and in the boundary condition optimization (section III.D) are detailed here. These transformations map 4D $(u, v, t, \tau)$ object coordinates onto unique 3D $(A, B, C)$ coordinates for each point in an m-rep figure and onto unique 2D $(a, b)$ coordinates for each point on the surface of an m-rep figure. The mappings from $(u, v, t, \tau)$ to $(A, B, C)$ and $(a, b)$ are analytically invertible, allowing direct conversion between any of the three coordinate spaces. These transformations are important because when point coordinates are iteratively optimized, reducing the dimensionality of the coordinates improves the efficiency and robustness of the optimization.

An m-rep figure is divided into five regions: a center region and four rim regions, labelled $R_{A}, R_{B}, R_{C}, R_{D}$ and $R_{E}$ in 


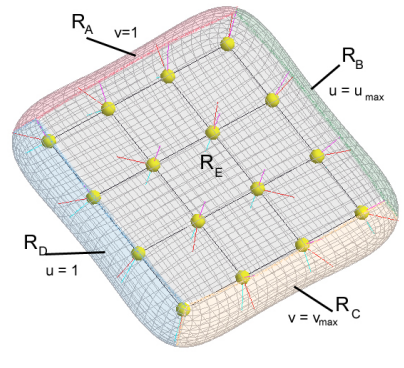

(a)

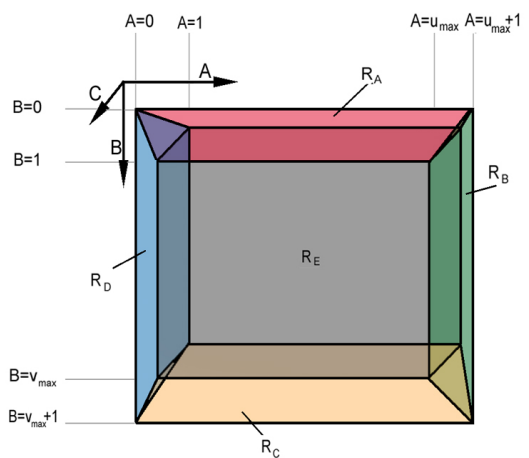

(b)

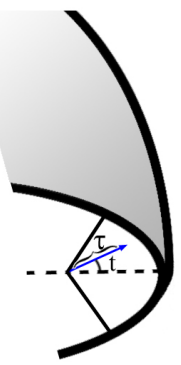

(c)

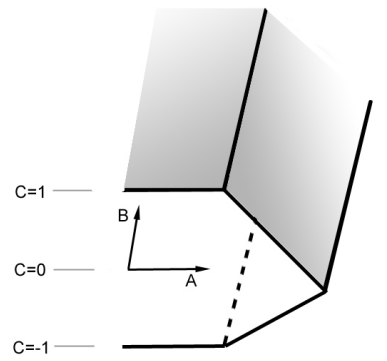

(d)

Fig. 17. (a) Five regions of an m-rep figure (b) Diagram of the $(A, B, C)$ parameter space with the five m-rep regions labelled (c) \& (d) A sliced view of one of the rim regions, with the polar type medial object coordinates shown on the left and the corresponding $(A, B, C)$ parameter space on the right.

Fig. 17 (a). In any single region only three of the four medial coordinates are needed to uniquely identify a point. For the entire center region, $R_{E}$, all points are referenced by $(u, v, 1, \tau) ; t$ does not vary. In each of the rim regions, $R_{A}, R_{B}, R_{C}$, and $R_{D}$, either $u$ or $v$ maintains a constant value. Therefore, the $(u, v, t, \tau)$ parameter space can be viewed as five three-dimensional parameter spaces rather than as one four-dimensional space. The $(A, B, C)$ parameter space simply maps these five three-dimensional segments into one continuous three-dimensional space. For the center region, the mapping is direct.

$$
A=u \quad B=v \quad C=\tau
$$

For the rim regions, a more complicated mapping is necessary because the $t$ and $\tau$ medial object coordinates for the rims are essentially polar coordinates, while the $(A, B, C)$ coordinates form a Cartesian type coordinate system. (See Fig. 17 (c) and (d)) The $(A, B, C)$ coordinates for each rim span a wedge shaped region, as shown in Fig. 17 (b). The equations for the $(u, v, t, \tau) \rightarrow(A, B, C)$ mapping for each rim region are as follows.

$$
\begin{aligned}
\alpha= & \frac{1}{\tan \left(\frac{t \pi}{2}\right)+1} \\
R_{A}: A= & v(1-|\tau| \alpha)+(1-v)(-|\tau| \alpha)\left(\frac{u_{\max }+1}{u_{\max }-1}\right) \\
B= & v \\
R_{B}: A= & u \\
B= & \left(u_{\max }+1-u\right)\left(v_{\max }+|\tau| \alpha\right) \\
& +\left(u-u_{\max }\right)\left(v_{\max }-1+|\tau| \alpha\right)\left(\frac{v_{\max }+1}{v_{\max }-1}\right) \\
R_{C}: A= & \left(v_{\max }+1-v\right)\left(u_{\max }+|\tau| \alpha\right) \\
& +\left(v-v_{\max }\right)\left(u_{\max }-1+|\tau| \alpha\right)\left(\frac{u_{\max }+1}{u_{\max }-1}\right) \\
B= & v \\
R_{D}: A= & u \\
B= & u(1-|\tau| \alpha)+(1-u)(-|\tau| \alpha)\left(\frac{v_{\max }+1}{v_{\max }-1}\right)
\end{aligned}
$$

$$
\begin{array}{r}
R_{A}, R_{B}, R_{C}, R_{D}: \\
C=\tau(1-\alpha)
\end{array}
$$

$(a, b)$ medial object surface coordinates are defined in terms of $(A, B, C)$ coordinates. The $(a, b)$ parameter space covers the surface of the $(A, B, C)$ parameter space, unfolded along the $\left(u_{\max }+1\right)$ edge.

$$
\begin{aligned}
& a= \begin{cases}A & \text { if } C \geq 0 \\
2\left(u_{\max }+1\right)-A & \text { if } C<0\end{cases} \\
& b=B
\end{aligned}
$$

\section{ACKNOWLEDGMENT}

We thank Gregg Tracton for assistance with image processing and Drs. Gilad Cohen, Nitin Mathur and William O'Meara for providing CT images and segmentations of the clinical prostate brachytherapy example.

\section{REFERENCES}

[1] A. Bharatha, M. Hirose, N. Hata, S. Warfield, M. Ferrant, K. Zou, E. Suarez-Santana, J. Ruiz-Alzola, A. D’Amico, R. Cormack, R. Kikinis, F. Jolesz, and C. Tempany, "Evaluation of three-dimensional finite element-based deformable registration of pre-and intraoperative prostate imaging," Medical Physics, vol. 28, pp. 2551-2560, Dec 2001.

[2] R. Alterovitz, K. Goldberg, J. Pouliot, I. J. Hsu, Y. Kim, S. M. Noworolski, and J. Kurhanewicz, "Registration of $\mathrm{mr}$ prostate images with biomechanical modeling and nonlinear parameter estimation," Medical Physics, vol. 33, no. 2, pp. 446-454, February 2006.

[3] A. Mohamed, C. Davatzikos, and R. H. Taylor, "A combined statistical and biomechanical model for estimation of intra-operative prostate deformation." in MICCAI (2), ser. Lecture Notes in Computer Science, T. Dohi and R. Kikinis, Eds., vol. 2489. Springer, 2002, pp. 452-460.

[4] O. Clatz, H. Delingette, I. F. Talos, A. J. Golby, R. Kikinis, F. A. Jolesz, N. Ayache, and S. K. Warfield, "Robust nonrigid registration to capture brain shift from intraoperative mri," IEEE Transactions on Medical Imaging, vol. 24, no. 11, pp. 1417-1427, 2005.

[5] M. Ferrant, A. Nabavi, B. Macq, F. A. Jolesz, R. Kikinis, and S. K. Warfield, "Registration of 3-D MR images of the brain using a finite element biomechanical model," IEEE Transactions on Medical Imaging, vol. 20, no. 12, pp. 1384-1397, 2001.

[6] S. K. Kyriacou, C. Davatzikos, S. J. Zinreich, and R. N. Bryan, "Nonlinear elastic registration of brain images with tumor pathology using a biomechanical model," IEEE Transactions on Medical Imaging, vol. 18 , no. 7, pp. 580-592, 1999. 
[7] A. Hagemann, K. Rohr, H. S. Stiehl, U. Spetzger, and J. M. Gilsbach, "Biomechanical modeling of the human head for physically based, nonrigid image registration," IEEE Transactions on Medical Imaging, vol. 18 , no. 10, pp. 875-884, October 1999.

[8] M. H. Xu and W. L. Nowinski, "Talairach-Tournoux brain atlas registration using a metalforming principle-based finite element method," Medical Image Analysis, vol. 5, pp. 271-279, 2001.

[9] F. Azar, D. N. Metaxas, and M. D. Schnall, "A deformable finite element model of the breast for predicting mechanical deformations under external perturbations," Journal of Academic Radiology, vol. 8, pp. 965-975, October 2001.

[10] A. Samani, J. Bishop, M. J. Yaffe, and D. B. Plewes, "Biomechanical 3-D finite element modeling of the human breast using mri data," IEEE Transactions on Medical Imaging, vol. 20, no. 4, pp. 271-279, April 2001.

[11] M. Chabanas and Y. Payan, "A 3d finite element model of the face for simulation in plastic and maxillo-facial surgery." in MICCAI, ser. Lecture Notes in Computer Science, S. L. Delp, A. M. DiGioia, and B. Jaramaz, Eds., vol. 1935. Springer, 2000, pp. 1068-1075.

[12] K. Brock, M. Sharpe, L. Dawson, S. Kim, and D. Jaffray, "Accuracy of finite element model-based multi-organ deformable image registration," Medical Physics, vol. 32, no. 6, pp. 1647-1659, 2005.

[13] D. M. Cash, M. I. Miga, T. K. Sinha, R. L. Galloway, and W. C. Chapman, "Compensating for intraoperative soft-tissue deformations using incomplete surface data and finite elements," IEEE Transactions on Medical Imaging, vol. 24, no. 11, pp. 1479-1491, November 2005.

[14] S. Cotin, H. Delingette, and N. Ayache, "Real-time elastic deformations of soft tissues for surgery simulation," IEEE Transactions On Visualization and Computer Graphics, vol. 5, no. 1, pp. 62-73, Jan-Mar 1999.

[15] J. A. Schnabel, C. Tanner, A. D. Castellano-Smith, A. Degenhard, M. O. Leach, D. R. Hose, D. L. G. Hill, and D. J. Hawkes, "Validation of nonrigid image registration using finite-element methods: application to breast mr images," IEEE Transactions on Medical Imaging, vol. 22, no. 2, pp. 238 - 247, February 2003.

[16] V. Narayana, P. L. Roberson, R. J. Winfield, and P. W. McLaughlin, "Impact of ultrasound and computed tomography prostate volume registration on evaluation of permanent prostate implants," International Journal of Radiation Oncology Biology Physics, vol. 39, no. 2, pp. 341346, 1997.

[17] M. V. Herk, A. Bruce, A. P. G. Kroes, T. Shouman, A. Touw, and J. V. Lebesque, "Quantification of organ motion during conformal radiotherapy of the prostate by three dimensional imgae registration," International Journal of Radiation Oncology Biology Physics, vol. 33, no. 5, pp. 1311-1320, 1995.

[18] B. W. Fei, C. Kemper, and D. L. Wilson, "A comparative study of warping and rigid body registration for the prostate and pelvic MR volumes," Computerized Medical Imaging and Graphics, vol. 27, no. 4, pp. 267-281, 2003.

[19] M. Hirose, A. Bharatha, N. Hatga, K. H. Zou, S. K. Warfield, R. A. Cormack, A. D'Amico, R. Kikinis, F. A. Jolesz, and C. M. Tempany, "Quantitative MR imaging assessment of prostate gland deformation before and during MR imaging-guided brachytherapy," Academic Radiology, vol. 9, no. 8, pp. 906-912, August 2002.

[20] S. E. Benzley, E. Perry, K. Merkley, B. Clark, and G. Sjaardama, "A comparison of all hexagonal and all tetrahedral finite element meshes for elastic and elasto-plastic analysis," Proceedings, 4th International Meshing Roundtable, pp. 179-191, October 1995.

[21] S. M. Pizer, J. Z. Chen, P. T. Fletcher, Y. Fridman, D. S. Fritsch, A. G. Gash, J. M. Glotzer, M. R. Jiroutek, S. Joshi, C. Lu, K. E. Muller, A. Thall, G. Tracton, P. Yushkevich, and E. L. Chaney, "Deformable m-reps for 3D medical image segmentation," International Journal of Computer Vision, vol. 55, no. 2/3, pp. 85-106, 2003.

[22] S. M. Pizer, P. T. Fletcher, S. Joshi, A. G. Gash, J. stough, A. Thall, G. Tracton, and E. L. Chaney, "A method \& software for segmentation of anatomic object ensembles by deformable m-reps," Medical Physics, vol. 32, no. 5, pp. 1335-1345, May 2005.

[23] S. Joshi, S. Pizer, P. Fletcher, P. Yuxhkevich, A. Thall, and J. Marron, "Multiscale deformable model segmentation and statistical shape analysis using medial descriptions," IEEE Transactions on Medial Imaging, vol. 21, no. 5, pp. 538-550, 2002.

[24] J. R. Crouch, S. M. Pizer, E. L. Chaney, and M. Zaider, "Medially based meshing with finite element analysis of prostate deformation." in MICCAI (1), ser. Lecture Notes in Computer Science, R. E. Ellis and T. M. Peters, Eds., vol. 2878. Springer, 2003, pp. 108-115.

[25] J. Crouch, Medial Techniques for Automating Finite Element Analysis. dissertation, UNC-Chapel Hill department of computer science, 2003. [Online]. Available: http://midag.cs.unc.edu/pubs/phdthesis/JCrouch03.pdf

[26] Department of Mechanics and Materials, Lund University, CALFEM, a finite element toolbox to MATLAB, Lund, Sweden, 1999. [Online]. Available: http://www.byggmek.lth.se/Calfem/Calman/pdfcalfem.pdf

[27] K. Bathe, Finite Element Procedures. New Jersey: Prentice-Hall, 1996.

[28] P. Knupp, "Hexahedral mesh untangling and algebraic mesh quality metrics," Proceedings, 9th International Meshing Roundtable, pp. 173183, October 2000.

[29] J. Lagarias, J. Reeds, M. Wright, and P. Wright, "Convergence properties of the Nelder-Mead simplex method in low dimensions," SIAM Journal of Optimization, vol. 9, no. 1, pp. 112-147, 1998.

[30] Y. Fung and P. Tong, Classical and Computational Solid Mechanics. World Scientific Publishing Company, 2001.

[31] P. G. Ciarlet, Mathematical Elasticity Volume 1: Three-dimensional elasticity. Amsterdam: Elsevier Science Pubishers, 1988

[32] H. Chui and A. Rangarajan, "A new algorithm for non-rigid point matching," IEEE Conference on Computer Vision and Pattern Recognition (CVPR), vol. 2, pp. 44-51, 2000.

[33] M. Foskey, B. Davis, L. Goyal, S. Chang, E. Chaney, N. Strehl, S. Tomei, J. Rosenman, and S. Joshi, "Large deformation three-dimensional image registration in image-guided radiation therapy," Phys Med Biol, vol. 50, pp. 5869-5892, 2005.

[34] B. Couteau, Y. Payan, and S. Lavallee, "The mesh-matching algorithm: an automatic 3D mesh generator for finite element structures," Journal of Biomechanics, vol. 33, pp. 1005-1009, 2000.

[35] R. Schneiders, "Quadrilateral and hexahedral element meshes," in Handbook of Grid Generation, J. F. Thompson, B. K. Soni, and N. P. Weatherill, Eds. Florida: CRC Press, 1998, pp. 21.1 - 21.27.

[36] Y. Fung, Biomechanics: Mechanical Properties of Living Tissues, 2nd ed. New York: Springer, 1993.

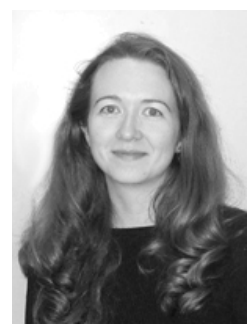

Jessica Crouch is an assistant professor of computer science at Old Dominion University in Norfolk, Virginia. She received a Ph.D. in computer science in 2003 and master's degree in computer science in 1999, both from from the University of North Carolina at Chapel Hill. Her Bachelor's degree, also in computer science, is from the University of Richmond. She completed a postdoc at the Engineering Research Center at The Johns Hopkins University in Baltimore, Maryland, and is involved in collaborative research with Memorial Sloan-Kettering Cancer

Center.

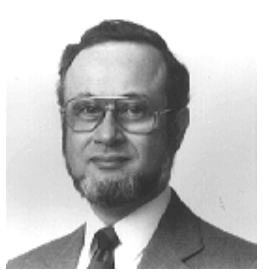

Stephen M. Pizer is Kenan Professor of Computer Science, Radiology, Radiation Oncology and Biomedical Engineering at the University of North Carolina, Chapel Hill. His Bachelor degrees are from Brown University, in Applied Mathematics, and his Ph.D. is from Harvard University in 1967, in Computer Science. Dr. Pizer heads UNCs multidepartmental Medical Image Display and Analysis Group, a collaborative research group of over 100 professionals. His recent research focus has been on medial models of the geometry of objects and multiobject complexes and the use of these models in 3D image segmentation and statistical characterization of object geometry. 
Edward L. Chaney is the head of the Physics and Computing Division of the Department of Radiation Oncology at the University of North Carolina at Chapel Hill. He received his $\mathrm{PhD}$ in physics from the University of Tennessee in 1969 and his Bachelor's in physics from Milsaps College in 1965. His research interests include the development of new techniques for image guided radiotherapy treatment planning and verification as well as translational research involving migration of imaging research into clinical systems. Current research is focused on deformable medial models for image segmentation to define normal anatomical structures; registration of volumetric images for tumor localization; and analysis of portal images to measure treatment field shape and to detect treatment setup errors.

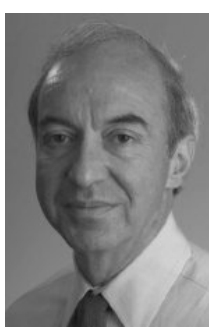

Gig S. Mageras is a medical physicist and acting chief of the Medical Physics Computer Service at Memorial Sloan-Kettering Cancer Center. His research interests are in radiation treatment planning and verification. Recent research has focused on volume imaging and registration techniques to improve tumor identification, and treatment planning methods to reduce positional uncertainty associated with internal organ motion. Dr. Mageras received his Ph.D. degree in physics from Columbia University in 1982 .
Yu-Chi Hu is a senior programmer/analyst of Department of Medical Physics at Memorial Sloan-Kettering Cancer Center and a PhD student in Computer Science at the Graduate Center at City University of New York. He received Computer Systems Engineer degree from Columbia University in 2003, M.S in Computer Science from State University of New York at Binghamton in 1994, and B.B.A. in Computer and Information Science from Soochow University in Taiwan in 1989.
Marco Zaider is the Head of Brachytherapy Physics at Memorial SloanKettering Hospital and Professor of Physics (Radiology) at Cornell Medical School. His research interests include microdosimetry, interaction of ionizing radiation with biomolecules (DNA), algorithms for treatment plan optimization and biophysical modeling of radiation effects. 